\title{
Use of N-nitrosodimethylamine (NDMA) contaminated valsartan products and risk of cancer: Danish nationwide cohort study
}

\section{FAST TRACK}

${ }^{1}$ Clinical Pharmacology and

Pharmacy, Department of Public

Health, University of Southern

Denmark, JB Winsløwsvej 19, 2

5000 Odense C, Denmark

${ }^{2}$ Medical Evaluation and

Biostatistics, Danish Medicines

Agency, Copenhagen, Denmark

${ }^{3}$ Pharmacovigilance and

Medical Devices, Danish

Medicines Agency,

Copenhagen, Denmark

Correspondence to: A Pottegård apottegaard@health.sdu.dk

Additional material is published online only. To view please visit the journal online.

Cite this as: BMJ 2018;362:k3851 http://dx.doi.org/10.1136/bmj.k3851

Accepted: 09 September 2018

\author{
Anton Pottegård, ${ }^{1}$ Kasper Bruun Kristensen, ${ }^{1}$ Martin Thomsen Ernst, ${ }^{1}$ Nanna Borup Johansen, ${ }^{2}$ \\ Pierre Quartarolo, ${ }^{3}$ Jesper Hallas ${ }^{1}$
}

\section{ABSTRACT}

\section{OBJECTIVE}

To perform an expedited assessment of cancer risk associated with exposure to $\mathrm{N}$-nitrosodimethylamine (NDMA) through contaminated valsartan products.

\section{DESIGN}

Nationwide cohort study.

\section{SETTING}

Danish health registries on individual level prescription drug use, cancer occurrence, and hospital diagnoses.

\section{PARTICIPANTS}

5150 Danish patients with no history of cancer, aged 40 years or older, and using valsartan at 1 January 2012 or initiating use between 1 January 2012 and 30 June 2017. Participants were followed from one year after cohort entry (lag time period) until experiencing a cancer outcome, death, migration, or end of study period (30 June 2018). Each participant's exposure to NDMA (ever exposure and predefined categories of cumulative valsartan exposure) was mapped out as a time varying variable while also applying a one year lag.

\section{MAIN OUTCOME MEASURES}

Association between NDMA exposure and a primary composite endpoint comprising all cancers except non-melanoma skin cancer, estimated using Cox regression. In supplementary analyses, the risk of individual cancers was determined.

RESULTS

The final cohort comprised 5150 people followed for a median of 4.6 years. In total, 3625 cohort participants contributed 7344 person years classified as unexposed to NDMA, and 3450 participants contributed 11920 person years classified as ever exposed to NDMA. With 104 cancer outcomes among NDMA unexposed participants and 198 among exposed participants, the adjusted hazard ratio for overall cancer was 1.09 (95\% confidence interval 0.85 to 1.41$)$, with no evidence

\section{WHAT IS ALREADY KNOWN ON THIS TOPIC}

Some valsartan products are suspected of having been contaminated with $\mathrm{N}$-nitrosodimethylamine (NDMA), which is classified as carcinogenic to humans After the discovery, European medical agencies and the US Food and Drug Administration withdrew affected valsartan products from the market

\section{WHAT THIS STUDY ADDS}

Among Danish valsartan users, exposure to NDMA contaminated valsartan was not associated with a markedly increased risk of overall cancer (adjusted hazard ratio $1.09,95 \%$ confidence interval 0.85 to 1.41 )

Future studies are, however, required to evaluate the risks for single cancer outcomes as well as long term effects of a dose-response relation $(\mathrm{P}=0.70)$. For single cancer outcomes, increases in risk were observed for colorectal cancer (hazard ratio 1.46, 95\% confidence interval 0.79 to 2.73 ) and for uterine cancer (1.81, 0.55 to 5.90 ), although with wide confidence intervals that included the null.

\section{CONCLUSIONS}

The results do not imply a markedly increased short term overall risk of cancer in users of valsartan contaminated with NDMA. However, uncertainty persists about single cancer outcomes, and studies with longer follow-up are needed to assess long term cancer risk.

\section{Introduction}

Valsartan is an angiotensin II receptor antagonist used to treat hypertension and heart failure. ${ }^{12}$ In July 2018 , some valsartan products were discovered to have been contaminated with N-nitrosodimethylamine (NDMA). ${ }^{3}$ This contamination, which far exceeded regulatory exposure limits, was specific to drug products manufactured by Zhejiang Huahai Pharmaceuticals, a company in Linhai, China, and seems to be related to a change in the manufacturing process that was implemented in 2012. Consequently, medical agencies across Europe as well as the US Food and Drug Administration have withdrawn all affected valsartan products from the market as of July $2018 .^{3}$

NDMA is the simplest dialkylnitrosamine and is known to be a by-product in various industries-for example, the manufacture of pesticides, rubber tyres, alkylamines, and dyes. ${ }^{4}$ NDMA is one of the most well characterised and most potent animal carcinogens known and has been shown to be a potent carcinogen across all species that have been investigated, both as single doses and with long term exposure to lower quantities. ${ }^{5}$ Although no in vivo data are available for humans, NDMA seems to be metabolised similarly in human tissue and rodent tissue. ${ }^{6}$ The International Agency for Research on Cancer (IARC) has on this basis classified NDMA as "probably carcinogenic to humans" (group 2A), emphasising that NDMA "should be regarded for practical purposes as if it were carcinogenic to humans."7

We accessed the nationwide Danish healthcare registries and conducted an expedited observational cohort study of the association between use of potentially NDMA contaminated valsartan products and risk of cancer. Our aim was to quantify the potential consequences of NDMA contaminated drug products entering the market and to provide timely information for regulatory bodies evaluating this potential public health issue. 


\section{Methods}

We conducted a cohort study comparing cancer outcomes in users of potentially NDMA contaminated valsartan products with users of valsartan products assumed free from this contaminant.

\section{Data sources and linkage}

We obtained data from four Danish nationwide registries: the Danish Cancer Registry, ${ }^{89}$ the National Prescription Registry, ${ }^{10}$ the National Patient Register, ${ }^{11}$ and the Civil Registration System. ${ }^{12}$ Supplementary appendix A describes the data sources in detail and appendix B provides the codes for cancer diagnoses, drug exposures, and covariates. Data were linked by the personal identification number, a unique identifier assigned to all Danish residents since $1968 .{ }^{13}$ Virtually all medical care in Denmark is provided by the national health authorities, allowing population based register linkage studies covering all legal residents of Denmark.

\section{Study cohort}

The study cohort comprised all Danish patients filling a valsartan prescription during the study period of 1 January 2012 to 30 June 2018. Prevalent users of valsartan at the start of the study period-defined as individuals having filled a valsartan prescription in September to the end of December 2011, entered the study cohort at 1 January 2012, whereas incident users entered the study cohort at the day of filling their first valsartan prescription during the study period. As patients contributed risk time from one year after entering the study cohort, we excluded those with less than one year of follow-up, as they did not contribute to any of the analyses reported. For the same reason, we excluded incident users filling their first prescription after 30 June 2017. We further excluded patients with a record of a previous cancer except non-melanoma skin cancer; those with a recent migration before cohort entry (within two years) to ensure enough baseline data on all study participants; and those aged less than 40 years at cohort entry as both use of valsartan and cancer occurrence is rare among children and younger adults. Participants were followed until a cancer outcome, death, migration, or end of the study period (30 June 2018), whichever occurred first.

\section{Ascertainment of NDMA exposure}

Within the study cohort we mapped out each participant's exposure to NDMA contamination using the unique drug ID (Nordic article number) as recorded in the National Prescription Registry to identify the single valsartan product and its manufacturer. From the 128 unique valsartan drug products used during 2012-18 within our study population, we identified 18 drug products (which constituted $18 \%$ of all prescriptions filled) that were manufactured using an active pharmaceutical ingredient from Zhejiang Huahai Pharmaceuticals. These drug products were classified as probably contaminated with NDMA. An additional 36 drug products ( $26 \%$ of all prescriptions) were classified as possibly contaminated with NDMA, as they contained an active pharmaceutical ingredient both from Zhejiang Huahai Pharmaceuticals and from other companies. Seventy four drug products (55\% of all prescriptions) were classified as unlikely to be contaminated with NDMA as they did not contain an active pharmaceutical ingredient from Zhejiang Huahai Pharmaceuticals. In the main analysis we pooled together valsartan prescriptions classified as probably and possibly contaminated with NDMA, classifying those filling such prescriptions as ever exposed to NDMA from their first occurrence of such a prescription. We further stratified NDMA exposed person time by cumulative dose from filled prescriptions of potentially NDMA containing valsartan tablets (applying preplanned stratums of $<20000,20000-49999$, and $\geq 50000 \mathrm{mg}$ ). The use of milligrams of valsartan as a scale for the doseresponse analysis was based on the observation that the NDMA content for each tablet seems to correlate with the strength of the tablet. ${ }^{14}$ With an estimated daily use of $80-160 \mathrm{mg}$ (the defined daily dose of valsartan is $80 \mathrm{mg}^{15}$ ), these cut-offs corresponded roughly to $<200,200-499$, and $\geq 500$ tablets. Of note, individuals classified as exposed to NDMA contributed follow-up to the non-exposed cohort until filling their first prescription for a potentially NDMA contaminated product. This ensured that the estimates were not affected by immortal time bias. ${ }^{16}$

Throughout all assessments of potential exposure to NDMA, we applied a one year lag time-that is, persons contributed NDMA exposed person time from one year after having filled their first prescription for a potentially NDMA containing valsartan product and onwards. This was done as very recent NDMA exposure ( $<1$ year) is considered unlikely to materially affect an individual's risk of receiving a cancer diagnosis. ${ }^{17}$ The length of the lag time was subjected to sensitivity analyses.

\section{Cancer outcomes}

We obtained cancer outcomes from the Danish Cancer Registry. ${ }^{89}$ However, as data in this registry is currently only updated to 2016, we used the Danish National Patient Registry ${ }^{11}$ to ascertain outcomes from 1 January 2017 to 30 June 2018. The primary outcome was a composite endpoint comprising all cancers (except non-melanoma skin cancer), as NDMA exposure is suspected to increase the risk of several different cancers. In supplementary analyses, we determined the risk of individual cancers, grouping cancers by organ system (ie, using codes from the international classification of diseases, 10th revision).

\section{Covariates}

The study cohort was described according to several characteristics that were also incorporated as covariates in the analyses: use of drugs (prescription fill $<120$ days before cohort entry) known or suspected to affect cancer risk, including low dose aspirin, nonaspirin non-steroidal anti-inflammatory drugs, 5- $\alpha$ reductase inhibitors, statins, spironolactone, oral 
steroids, hormone replacement therapy, and selective serotonin reuptake inhibitors ${ }^{18}$; prior diagnoses (within five years from cohort entry) of diabetes, chronic obstructive pulmonary disease, heart failure, and alcohol related disease; Charlson comorbidity index scores (0, low; $1-2$, medium; or $\geq 3$, high; based on diagnoses established within the past five years before cohort entry ${ }^{1920}$; and whether the participant was a prevalent valsartan user at the beginning of the study period or initiated valsartan during the study period.

\section{Main analysis}

The primary analysis comprised a comparison of cancer occurrence during follow-up exposed to NDMA versus follow-up not exposed to NDMA. We used Cox regression to estimate the hazard ratio with $95 \%$ confidence intervals for cancer associated with NDMA exposure, both for ever use and for the predefined categories of cumulative use. The proportional hazards assumption was tested using Schoenfeld residuals. We carried out a formal dose-response test by categorising cumulative exposure to NDMA contaminated valsartan in categories of $10000 \mathrm{mg}$ as a time varying exposure and obtaining the $\mathrm{P}$ value for this variable as a continuous predictor of cancer risk in a Cox regression. As all comparisons were performed within users of valsartan, the exposure to NDMA can reasonably be expected to be a random event, and confounding is thus expected to be limited. Analyses were, however, performed as crude comparisons adjusted only for sex and age (age at cohort entry as continuous variable) as well as adjusted for sex, age, and the potential confounding factors. All analyses were performed using STATA Release 15.2.

\section{Sensitivity and supplementary analyses}

We carried out several sensitivity and supplementary analyses. Firstly, we performed analyses stratifying all participants by sex and age (40-69 and $\geq 70$ years at cohort entry). Secondly, we restricted the cohort to prevalent valsartan users at the start of the study and to incident users during the study period. Thirdly, we restricted the ascertainment of NDMA exposure to prescriptions classified as probably contaminated with NDMA, while censoring individuals filling a prescription for a possibly NDMA contaminated drug product from the reference cohort (although allowing them to later enter the NDMA exposed cohort). Lastly, we varied the one year lag time period applied in the main analysis to six months and two years.

\section{Patient and public involvement}

No patients were involved in setting the research question or the outcome measures, nor were they involved in developing plans for design or implementation of the study. No patients were asked to advise on interpretation or writing of results. There are no plans to disseminate the results of the research directly to the patient community. However, the results will be included in the ongoing review of the potential impact of NDMA contaminated valsartan on patients by the European Medicines Agency.

\section{Results}

In initial descriptive analyses, we identified 7068 unique individuals filling a total of 95650 valsartan prescriptions from January 2012 to June 2018, the period where NDMA contaminated products were on the Danish market. The overall use of valsartan increased slightly during this period, in particular in 2017 and 2018 (fig 1), and the use of valsartan products possibly or probably contaminated with NDMA constituted about half of the total valsartan use, although this proportion dropped slightly during 2017-18.

For the selection of the study cohort, we identified 6406 individuals filling a valsartan prescription between September 2011 and June 2017. Of these, 5150 unique individuals met our inclusion criteria and entered the final cohort (fig 2), contributing a median of 4.6 years (interquartile range 2.0-5.5 years) of follow-up to the analysis, after the application of a one year lag period. Table 1 includes the baseline characteristics of valsartan users entering the study. A total of 3625 participants contributed 7344 person years of follow-up classified as unexposed to NDMA, and 3450 participants contributed 11920 person years classified as ever exposed to NDMA (fig 2). The distribution of potentially NDMA contaminated and non-contaminated prescriptions were similar between the study cohort and all valsartan users (see supplementary figure 1).

Overall, exposure to potentially (probably or possibly) NDMA contaminated valsartan products showed no association with cancer compared with exposure to valsartan products unlikely to be contaminated with NDMA (adjusted hazard ratio 1.09, $95 \%$ confidence interval 0.85 to 1.41 ) and no evidence of a dose-response relation $(\mathrm{P}=0.70$, table 2$)$.

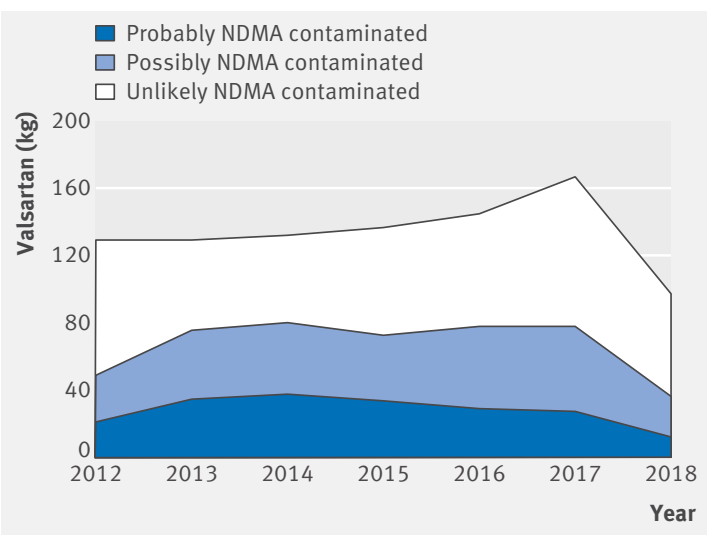

Fig 1 | Use of valsartan in kilograms of active substance, specified by drug products classified as probably, possibly, or unlikely to be contaminated with $\mathrm{N}$-nitrosodimethylamine (NDMA). The drop in 2018 results from data only being available to June 2018 


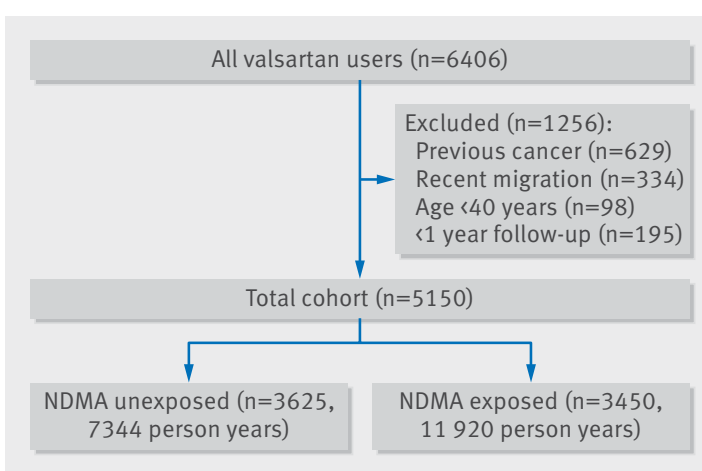

Fig 2 | Flowchart of cohort selection of Danish users of valsartan, January 2012 to June 2018. NDMA=Nnitrosodimethylamine

In analyses of single cancer outcomes, increased risks were seen for colorectal cancer (hazard ratio 1.46, 95\% confidence interval 0.79 to 2.73 ) and for uterine cancer $(1.81,0.55$ to 5.90$)$, although neither these nor other single cancer outcomes reached statistical significance (fig 3). Analyses of other cancer outcomes were not possible owing to low numbers-that is, no cancer outcomes outside those included in figure 3 showed any associations with NDMA use.

Results comparable to the main analyses were found when we stratified by sex and age, whereas a stronger association was seen when we restricted to incident users during the study period (hazard ratio $1.58,95 \%$ confidence interval 0.99 to 2.52 ) compared with prevalent users at the beginning of the study period (0.91, 0.66 to 1.25) (fig 4). A test for interaction between being an incident valsartan user and the effect of exposure to NDMA yielded a p value of 0.059 .

The sensitivity analysis censoring individuals filling a prescription for a possibly NDMA contaminated valsartan product from the reference category yielded results comparable to those of the main analyses, both for overall cancer (see supplementary table 1) and for single cancers (see supplementary figure 2).

Varying the lag time from one year used in the main analyses to six months or two years yielded slightly higher risk estimates with increasing lag time, with the hazard ratio for ever exposure increasing to 1.17 (95\% confidence interval 0.88 to 1.55 ) when a two year lag time was applied, although this did not reach statistical significance (see supplementary table 2).

\section{Discussion}

In this nationwide cohort study of Danish valsartan users, we did not see an increased short term overall risk of cancer associated with the use of valsartan products potentially contaminated with N-nitrosodimethylamine (NDMA).

\begin{tabular}{|c|c|c|c|}
\hline \multirow[t]{2}{*}{ Characteristics } & \multirow[t]{2}{*}{ All $(n=5150)$} & \multicolumn{2}{|l|}{ NDMA exposure } \\
\hline & & Exposed* $(n=3450)$ & Not exposed* $(n=3625)$ \\
\hline \multicolumn{4}{|l|}{ Sex: } \\
\hline Men & $2531(49.1)$ & $1630(46.9)$ & $1745(43.6)$ \\
\hline Women & $2619(50.9)$ & $1820(53.1)$ & $1880(56.4)$ \\
\hline \multicolumn{4}{|l|}{ Age (years): } \\
\hline Median (interquartile range) & $66(58-74)$ & - & - \\
\hline $40-69$ & $3195(62.0)$ & $2197(65.0)$ & $2164(61.2)$ \\
\hline$\geq 70$ & $1955(38.0)$ & $1253(35.0)$ & $1461(38.8)$ \\
\hline \multicolumn{4}{|l|}{ Prevalent valsartan userst: } \\
\hline No & $2870(55.7)$ & $2012(51.2)$ & $1353(25.7)$ \\
\hline Yes & $2280(44.3)$ & $1438(48.8)$ & $2272(74.3)$ \\
\hline \multicolumn{4}{|l|}{ Charlson comorbidity score: } \\
\hline 0 (low) & $3864(75.0)$ & $2697(79.0)$ & $2635(74.9)$ \\
\hline 1 & $884(17.2)$ & $541(15.3)$ & $670(17.1)$ \\
\hline 2 & $217(4.2)$ & $117(3.2)$ & $168(4.5)$ \\
\hline$\geq 3$ (high) & $185(3.6)$ & $95(2.5)$ & $152(3.4)$ \\
\hline \multicolumn{4}{|l|}{ Drugs: } \\
\hline Low dose aspirin & $1388(27.0)$ & $842(25.2)$ & $1092(29.2)$ \\
\hline Non-aspirin NSAID & $772(15.0)$ & $533(15.5)$ & $513(16.0)$ \\
\hline Statins & $1924(37.4)$ & $1185(35.1)$ & $1457(37.4)$ \\
\hline Spironolactone & $405(7.9)$ & $117(3.2)$ & $362(4.9)$ \\
\hline Glucocorticoids for systemic use & $244(4.7)$ & $166(4.5)$ & $171(4.3)$ \\
\hline 5- $\alpha$ reductase inhibitors & $64(1.2)$ & $41(1.2)$ & $47(0.9)$ \\
\hline SSRIS & $299(5.8)$ & $196(5.7)$ & $223(6.0)$ \\
\hline Hormone replacement therapy & $454(8.8)$ & $319(9.8)$ & $338(9.9)$ \\
\hline \multicolumn{4}{|l|}{ Diagnoses: } \\
\hline Diabetes type 1 and 2 & $899(17.5)$ & $559(16.1)$ & $667(18.0)$ \\
\hline Chronic obstructive pulmonary disease & $247(4.8)$ & $131(3.5)$ & $200(4.3)$ \\
\hline Congestive heart failure & $535(10.4)$ & $117(2.9)$ & $497(5.3)$ \\
\hline Alcohol related disease & $48(0.9)$ & $28(0.7)$ & $34(0.7)$ \\
\hline \multicolumn{4}{|c|}{$\begin{array}{l}\text { NSAID=non-steroidal anti-inflammatory drug; SSRIs=selective serotonin reuptake inhibitors. } \\
\text { *Characteristics weighted by proportion of total time exposed or not exposed that individuals contributed, thereby providing the distribution of covariate } \\
\text { in the main analysis comparison. } \\
\text { tDefined as being included in the study at } 1 \text { January } 2012 \text { by having filled a valsartan prescription between September and December } 2011 \text {. }\end{array}$} \\
\hline
\end{tabular}




\begin{tabular}{|c|c|c|c|c|c|}
\hline NDMA exposure & $\begin{array}{l}\text { Follow-up } \\
\text { (person years) }\end{array}$ & $\begin{array}{l}\text { Cancer } \\
\text { outcomes }\end{array}$ & $\begin{array}{l}\text { Incidence rate } \\
\text { (/1000 person years) }\end{array}$ & $\begin{array}{l}\text { Adjusted hazard ratio* } \\
(95 \% \mathrm{Cl})\end{array}$ & $\begin{array}{l}\text { Fully adjusted hazard } \\
\text { ratiot }(95 \% \mathrm{Cl})\end{array}$ \\
\hline Never use & 7344 & 104 & 14.2 & 1.00 (ref) & 1.00 (ref) \\
\hline Ever exposure & 11920 & 198 & 16.6 & $1.16(0.91$ to 1.49$)$ & $1.09(0.85$ to 1.41$)$ \\
\hline \multicolumn{6}{|c|}{ Cumulative exposure (mg)‡: } \\
\hline 20000-49999 & 2836 & 44 & 15.5 & $1.07(0.75$ to 1.53$)$ & $0.99(0.69$ to 1.43$)$ \\
\hline$\geq 50000$ & 5308 & 87 & 16.4 & $1.14(0.84$ to 1.54$)$ & $1.11(0.82$ to 1.50$)$ \\
\hline Test for trend§ & & & & $P=0.65$ & $P=0.70$ \\
\hline \multicolumn{6}{|c|}{$\begin{array}{l}\text { *Adjusted for age and sex. } \\
\text { †Adjusted for sex, age, use of low dose aspirin, non-aspirin non-steroidal anti-inflammatory drugs, } 5 \text { - } \alpha \text { reductase inhibitors, statins, spironolactone, oral } \\
\text { steroids, hormone replacement therapy, or selective serotonin reuptake inhibitors, history of diabetes, chronic obstructive pulmonary disease, heart } \\
\text { failure, or alcohol related disease, Charlson comorbidity index score, and being a prevalent valsartan user. } \\
\text { ‡Defined by total amount of NDMA contaminated valsartan filled. } \\
\text { §Estimated using Cox regression across } 10000 \mathrm{mg} \text { stratums of NDMA contaminated valsartan filled. }\end{array}$} \\
\hline
\end{tabular}

\section{Strengths and limitations of this study}

The principal strength of this study is the use of high quality nationwide registries, ${ }^{9}{ }^{10} 11$ leaving little potential for selection bias. ${ }^{12}$ Furthermore, the use of dispensing data, instead of data on prescribed drugs, as a proxy for NDMA exposure reduces the risk of misclassification due to primary non-adherence. ${ }^{21}$ The principal weakness of the study is the limited median follow-up. Our findings only pertain to early cancer risk after exposure to NDMA whereas future studies are needed to elucidate the total cancer risk, which requires a substantially longer follow-up for the individual than what is currently available. Additionally, the limited follow-up combined with the low use of valsartan in Denmark leads to limited precision. Lastly, our exposure ascertainment is based on assumptions about NDMA content. Reassuringly, our sensitivity analysis disregarding less certain sources of NDMA returned estimates comparable to those of the main analysis. However, future studies should utilise data on the actual NDMA content of individual valsartan tablets once such information becomes available.

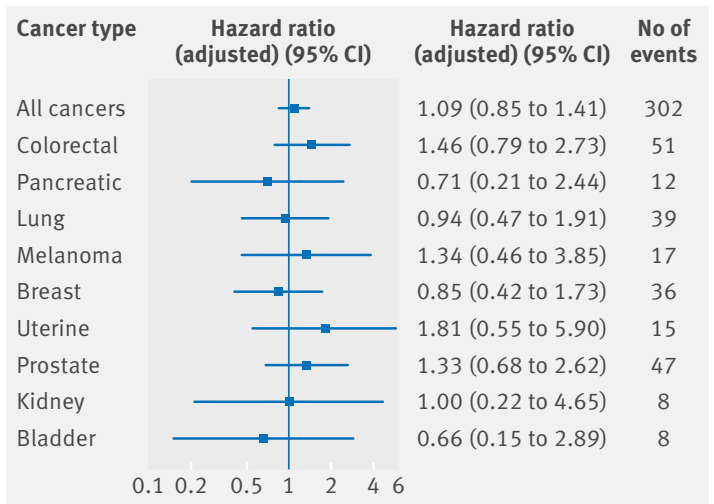

Fig 3 | Estimates for association between use of potentially N-nitrosodimethylamine (NDMA) contaminated valsartan products and risk of single cancer outcomes compared with users of noncontaminated valsartan products. Number of events are total number of events among valsartan users

\section{Biological rationale}

The International Agency for Research on Cancer (IARC) has classified NDMA as "probably carcinogenic to humans" owing to limited evidence of carcinogenicity in humans and sufficient evidence of carcinogenicity in animal studies. ${ }^{7}$ NDMA is suspected to have both localised and systemic carcinogenic effects due to the induction of DNA-damaging metabolites in the gastrointestinal tract and liver. ${ }^{622}$ Specifically, in the liver, NDMA is metabolised by CYP2E1 to methyldiazonium, which causes mutations by methylation. ${ }^{23}$ Also, N-nitroso compounds such as NDMA activate ras oncogenes, which are thought to play a role in the development of colon cancer. ${ }^{6}$ As such, tumours in the gastrointestinal tract, lungs, kidneys, and liver have been seen in animal studies. ${ }^{5} 2324$ Evidence of carcinogenicity in rats was found at doses of about $10 \mu \mathrm{g} / \mathrm{kg} / \mathrm{day} .{ }^{23}$ With concentrations of up to $22 \mu \mathrm{g}$ NDMA in $320 \mathrm{mg}$ valsartan tablets and $10 \mu \mathrm{g}$ NDMA in $160 \mathrm{mg}$ tablets, ${ }^{14}$ the daily exposure for a $70 \mathrm{~kg}$ person ranges from 0.14 to $0.31 \mu \mathrm{g} / \mathrm{kg} /$ day. Even though it is not possible to extrapolate directly from animals to humans, the daily exposure in humans is thus roughly 30 times lower than the lowest dose leading to liver cancer in rats. Owing to the known carcinogenic effect of NDMA in animals,



Fig 4 | Estimates for association between use of potentially $\mathrm{N}$-nitrosodimethylamine (NDMA) contaminated valsartan products and cancer risk compared with users of non-contaminated valsartan products, specified by patient subgroups. Number of events are total number of events among valsartan users 
no experimental studies in humans exist. However, as some dietary products (eg, processed meat) are known to contain small amounts of NDMA, epidemiological studies based on food frequency questionnaire data have been performed. Even though such studies are highly prone to confounding, three found an increased risk of gastrointestinal cancer with exposure to NDMA, predominantly colorectal cancer. ${ }^{25} 2627$ This finding, together with that from the animal studies, provides some support for the increased although statistically non-significant risk for this particular cancer observed in our study. Only one previous paper has reported on uterine cancer, finding no association between exposure to NDMA and uterine cancer in rats. ${ }^{28}$ Lastly, no estimates could be obtained for liver cancer in our study owing to the absence of liver cancer events among those exposed to NDMA. A markedly increased risk of liver cancer associated with NDMA exposure thus seems unlikely.

\section{Principal findings}

Our estimates pertain to early cancer risk associated with exposure to NDMA through contaminated valsartan products and should not be interpreted as evidence against NDMA being carcinogenic to humans in general. At most, our findings suggest that the levels of NDMA exposure achieved through valsartan products do not translate into a substantially increased short term cancer risk. Furthermore, the fact that our study evaluates a potential safety concern holds some implications about how to interpret the results. While the estimate for our primary outcome suggests a negligible and statistically non-significant increase in cancer risk of $9 \%$, it might be argued that a more cautious interpretation, reflecting the nature of the study question, would be to consider the upper limit of the confidence interval. Doing so leads to the different, although related, conclusion that we can reasonably exclude a more than $40 \%$ increased short term risk of cancer from exposure to NDMA contaminated valsartan products. A similar interpretation of the estimates obtained for the single cancer outcomes-in particular colorectal and uterine cancer-clearly highlights that our study cannot confidently rule out an increased risk from exposure to NDMA.

The finding that exposure to NDMA was associated with an increased risk of cancer specifically among users initiating valsartan treatment during the study period, as opposed to among valsartan users prevalent at the beginning of the study period, was a surprising finding that we cannot explain. The duration of followup was on average longer for prevalent users, as they were followed from the beginning of the study period (1 January 2012), and a late effect of exposure to NDMA therefore cannot explain this finding, as it would have led to an increased risk specifically among prevalent and not incident valsartan users. Considering the uncertainty about the actual NDMA content of valsartan products, it could be speculated that those using valsartan later in the study period might have been exposed to NDMA more often. However, no data are available that can be used to test this hypothesis. Lastly, our subgroup analyses had limited power and therefore the possibility of our results being a chance finding should also be considered.

\section{Policy implications}

Our findings can support regulators in their evaluation of the potential public health impact of exposure to NDMA through valsartan products. The Danish nationwide health registries and the strong research infrastructure hosted by Statistics Denmark and the Danish Health Data Authority, the latter of which was used in this study, gives researchers and regulators a unique possibility to provide answers to such emerging public health concerns in a timely manner. The present analysis was completed and submitted for publication within seven weeks after the finding of NDMA in valsartan products was announced publicly, and the paper published in The BMJ after a fast track peer review process spanning only three weeks from submission to publication. We previously performed a similar expedited assessment of a putative bleeding risk associated with use of generic warfarin, ${ }^{29} 30$ although its publication was delayed by the peer review process for several months. Besides rapid peer review assessment, a close collaboration between researchers and regulators is a key element in ensuring both speed and relevance of such research projects. In addition to knowledge about the risks associated with exposure to NDMA, the present study provides proof-of-concept for such processes, which hold great promise for the use of pharmacoepidemiological input in the regulatory assessment of future public health crises.

\section{Conclusion}

We have assessed the potential cancer risk associated with exposure to NDMA through contaminated valsartan products and found no evidence of a markedly increased short term overall risk of cancer. However, we cannot exclude a modest association. Furthermore, owing to the limited follow-up, assessment of long term effects was not possible, and the low number of events makes interpretation of estimates for single cancer outcomes difficult. Therefore, further studies are needed to fully elucidate the health effects of NDMA contaminated valsartan products.

We thank the Danish Health Data Authority, in particular Anna Birkmose Andersen and Anders Schierup, for providing expedited access to the registry data used in the study; Nicolai C Brun (Danish Medicines Agency) for valuable comments on the interpretation of the findings; and Camilla Artebjerg Bæk (Danish Medicines Agency) for providing toxicological input.

Contributors: $\mathrm{AP}, \mathrm{JH}, \mathrm{PQ}$, and $\mathrm{NB}$ J conceived and designed the study. AP, KBK, and MTE performed the statistical analyses and data management. AP and KBK drafted the initial manuscript. All authors interpreted the data and revised the manuscript critically. AP is the guarantor. The corresponding author attests that all listed authors meet authorship criteria and that no others meeting the criteria have been omitted.

Funding: None.

Competing interests: All authors have completed the ICMJE uniform disclosure form at www.icmje.org/coi_disclosure.pdf and declare: no support from any organisation for the submitted work; no financial 
relationships with any organisations that might have an interest in the submitted work in the previous three years; no other relationships or activities that could appear to have influenced the submitted work. Ethical approval: Not required.

Data sharing: Statistical code is available from AP upon request. No additional data are available as Danish legislation does not allow disclosure of individual level data.

Transparency: The lead author (AP) affirms that this manuscript is an honest, accurate, and transparent account of the study being reported; that no important aspects of the study have been omitted; and that any discrepancies from the study as planned have been explained.

This is an Open Access article distributed in accordance with the Creative Commons Attribution Non Commercial (CC BY-NC 4.0) license, which permits others to distribute, remix, adapt, build upon this work non-commercially, and license their derivative works on different terms, provided the original work is properly cited and the use is noncommercial. See: http://creativecommons.org/licenses/by-nc/4.0/.

1 Mancia G, Fagard R, Narkiewicz K, et al, Task Force Members. 2013 ESH/ESC Guidelines for the management of arterial hypertension: the Task Force for the management of arterial hypertension of the European Society of Hypertension (ESH) and of the European Society of Cardiology (ESC). J Hypertens 2013;31:1281-357. doi:10.1097/01.hjh.0000431740.32696.cc

2 Ponikowski P, Voors AA, Anker SD, et al, ESC Scientific Document Group. 2016 ESC Guidelines for the diagnosis and treatment of acute and chronic heart failure: The Task Force for the diagnosis and treatment of acute and chronic heart failure of the European Society of Cardiology (ESC)Developed with the special contribution of the Heart Failure Association (HFA) of the ESC. Eur Heart / 2016;37:2129-200. doi:10.1093/eurheartj/ehw128

3 European Medicines Agency. EMA reviewing medicines containing valsartan from Zhejiang Huahai following detection of an impurity. 2018. http://www.ema.europa.eu/ema/index.jsp?curl=pages/ news_and_events/news/2018/07/news_detail_002984. jsp\&mid=WC0b01ac058004d5c1. Last accessed 24/08/2018.

4 Priority Substances List Assessment Report for N-Nitrosodimethylamine (NDMA) Canadian Environmental Protection Act, 1999.

5 Agency for Toxic Substances and Disease Registry U.S. Public Health Service. Toxicological profile for N-nitrosodimethylamine. 1989. https://www.atsdr.cdc.gov/toxprofiles/tp141.pdf. Last accessed: 24/08/2018.

6 Tricker AR, Preussmann R. Carcinogenic N-nitrosamines in the diet: occurrence, formation, mechanisms and carcinogenic potential. Mutat Res 1991;259:277-89. doi:10.1016/0165-1218(91)90123-4

7 International Agency for Research on Cancer. IARC monographs on the evaluation of carcinogenic risks to humans. Overall evaluations of carcinogenicity: an updating of IARC Monographs Volumes 1-42. Lyon, France, 1987 supplement no 7.

8 Storm HH, Michelsen EV, Clemmensen IH, Pihl I. The Danish Cancer Registry--history, content, quality and use. Dan Med Bull 1997;44:535-9.

9 Gjerstorff ML. The Danish Cancer Registry. Scand J Public Health 2011;39(Suppl):42-5. doi:10.1177/1403494810393562

10 Pottegård A, Schmidt SAJ, Wallach-Kildemoes H, Sørensen HT, Hallas I, Schmidt M. Data Resource Profile: The Danish National Prescription Registry. Int J Epidemiol 2017;46:798-798f. doi:10.1093/ije/dyw213

11 Schmidt M, Schmidt SAI, Sandegaard IL, Ehrenstein V, Pedersen L, Sørensen HT. The Danish National Patient Registry: a review of content, data quality, and research potential. Clin Epidemiol 2015;7:449-90. doi:10.2147/CLEP.S91125

12 Schmidt M, Pedersen L, Sørensen HT. The Danish Civil Registration System as a tool in epidemiology. Eur J Epidemiol 2014;29:541-9. doi:10.1007/s10654-014-9930-3

13 Pedersen CB. The Danish Civil Registration System. Scand J Public Health 2011;39(Suppl):22-5. doi:10.1177/1403494810387965
14 Abdel-Tawab M, Gröner R, Kopp T, Meins J, Wübert J. ZL findet NDMA in Tabletten. PZ Pharm Ztg 2018;30. https://www. pharmazeutische-zeitung.de/index.php?id=77660

15 WHO Collaborating Centre for Drug Statistics Methodology. Guidelines for ATC classification and DDD assignment 2016. Oslo, 2016.

16 Weberpals J, Jansen L, van Herk-Sukel MPP, et al. Immortal time bias in pharmacoepidemiological studies on cancer patient survival: empirical illustration for beta-blocker use in four cancers with different prognosis. Eur J Epidemiol 2017;32:1019-31. doi:10.1007/s10654-017-0304-5

17 Pottegård A, Friis S, Stürmer T, Hallas J, Bahmanyar S. Considerations for Pharmacoepidemiological Studies of Drug-Cancer Associations. Basic Clin Pharmacol Toxicol 2018;122:451-9. doi:10.1111/bcpt.12946

18 Friis S, Kesminiene A, Espina C, Auvinen A, Straif K, Schüz J. European Code against Cancer 4th Edition: Medical exposures, including hormone therapy, and cancer. Cancer Epidemiol 2015;39(Suppl 1):S107-19. doi:10.1016/j.canep.2015.08.003

19 Charlson ME, Pompei P, Ales KL, MacKenzie CR. A new method of classifying prognostic comorbidity in longitudinal studies: development and validation. J Chronic Dis 1987;40:373-83. doi:10.1016/0021-9681(87)90171-8

20 Thygesen SK, Christiansen CF, Christensen S, Lash TL, Sørensen HT. The predictive value of ICD-10 diagnostic coding used to assess Charlson comorbidity index conditions in the populationbased Danish National Registry of Patients. BMC Med Res Methodol 2011;11:83. doi:10.1186/1471-2288-11-83

21 Pottegård A, Christensen Rd, Houji A, et al. Primary nonadherence in general practice: a Danish register study. Eur $/$ Clin Pharmacol 2014;70:757-63. doi:10.1007/s00228-014-1677-y

22 Bartsch H, K O'Neill I. Ninth International Meeting on N-Nitroso Compounds: Exposures, Mechanisms, and Relevance to Human Cancer. Cancer Res 1988;48:4711-4.

23 Peto R, Gray R, Brantom P, Grasso P. Nitrosamine carcinogenesis in 5120 rodents: chronic administration of sixteen different concentrations of NDEA, NDMA, NPYR and NPIP in the water of 4440 inbred rats, with parallel studies on NDEA alone of the effect of age of starting (3, 6 or 20 weeks) and of species (rats, mice or hamsters) IARC Sci Publ 1984;(57):627-65.

24 Lijinsky W, Reuber MD. Carcinogenesis in rats by nitrosodimethylamine and other nitrosomethylalkylamines at low doses. Cancer Lett 1984;22:83-8. doi:10.1016/0304-3835(84)90047-8

25 Loh YH, Jakszyn P, Luben RN, Mulligan AA, Mitrou PN, Khaw K-T. $\mathrm{N}$-Nitroso compounds and cancer incidence: the European Prospective Investigation into Cancer and Nutrition (EPIC)Norfolk Study. Am J Clin Nutr 2011;93:1053-61. doi:10.3945/ ajcn.111.012377

26 Zhu Y, Wang PP, Zhao J, et al. Dietary N-nitroso compounds and risk of colorectal cancer: a case-control study in Newfoundland and Labrador and Ontario, Canada. Br J Nutr 2014;111:1109-17. doi:10.1017/S0007114513003462

27 Knekt P, Järvinen R, Dich J, Hakulinen T. Risk of colorectal and other gastro-intestinal cancers after exposure to nitrate, nitrite and $\mathrm{N}$-nitroso compounds: a follow-up study. Int/ Cancer 1999;80:852-6. doi:10.1002/(SICI)10970215(19990315)80:6<852::AID-IIC9>3.0.CO;2-S

28 Peto R, Gray R, Brantom P, Grasso P. Effects on 4080 rats of chronic ingestion of $\mathrm{N}$-nitrosodiethylamine or $\mathrm{N}$-nitrosodimethylamine: a detailed dose-response study. Cancer Res 1991:51:6415-51.

29 Hellfritzsch M, Rathe J, Stage TB, et al. Generic switching of warfarin and risk of excessive anticoagulation: a Danish nationwide cohort study. Pharmacoepidemiol Drug Saf 2016;25:336-43. doi:10.1002/pds.3942

30 Heisterberg J. Automatic generic switching of warfarin: to do or not to do? Pharmacoepidemiol Drug Saf 2016;25:344-5. doi:10.1002/pds.3969

Supplementary information: appendices A and B 